\title{
Micro- and nanocoordinate measurements of micro-parts with 3-D tunnelling current probing
}

\author{
A. Schuler, T. Hausotte, and Z. Sun \\ Institute of Manufacturing Metrology, Friedrich-Alexander-Universität Erlangen-Nürnberg, Naegelsbachstr. 25, \\ 91052 Erlangen, Germany \\ Correspondence to: A. Schuler (alexander.schuler@fau.de)
}

Received: 28 November 2014 - Revised: 13 April 2015 - Accepted: 28 April 2015 - Published: 2 June 2015

\begin{abstract}
Measurement tasks of modern micro- and nanometrology are posing a problem for current measurement instruments with decreasing structure sizes and rising aspect ratios. There is an open requirement for nanometre-resolving 3-D capable sensors and corresponding 3-D positioning systems to operate the sensors for 3-D measurements. A 3-D probing system based on electrical interaction is presented which is operated on a nanopositioning system type SIOS NMM-1. Furthermore, we demonstrate the progress and new possibilities for 3-D measurements with the nanopositioning and nanomeasurement machine NMM-1 and also with the application of a rotary kinematic chain. In addition new 3-D measurement routines for the NMM-1, also for micro-tactile probing systems as well as current plans, are shown.
\end{abstract}

\section{Introduction}

The progress in micro- and nanometrology was always driven by the developments in manufacturing technology. With newer manufacturing possibilities enabling smaller structures and components with higher complexity, a corresponding improvement in metrology is required (Hansen et al., 2006). The measurement uncertainty has to be 5 to 10 times better than the required manufacturing tolerance (Berndt et al., 1968). This also applies to the uncertainty of the probing sensors for coordinate measuring systems. Modern measurement tasks require a continuous increase of the measurement systems' resolution. Also, the aspect ratio and the three-dimensionality of workpieces are rising with surface angles up to $90^{\circ}$. Examples for such workpieces would be cutting tools for micro-manufacturing. Micro-cutting tools, such as micro-drills, show tapered cutting edges with radii of single micro-metres combined with drill diameters down to $200 \mu \mathrm{m}$. As the shape of the cutting edge is essential for the cutting process and as for micro-tools a homogeneous sharp edge is very difficult to manufacture, the position and shape of the edge has to be measured (Bissacco et al., 2008; Hansen et al., 2006). Other measurement tasks cover micro-components like the micro-gears depicted in Fig. 1. These parts, either manufactured by cutting pro- cesses or the new technology of LIGA manufacturing show high aspect ratios with poorly accessible corners (Hansen et al., 2006).

The list of challenging components can be extended ranging from optical parts, such as micro-lenses or aspherics, to functional surfaces or micro-fluidic devices (Jiang and Whitehouse, 2012; Weckenmann et al., 2011). Generalizing, a resolution and uncertainty in the nanometre range and full 3 -D capability would be required. In contrast current sensors cannot completely meet these requirements, especially the necessary 3-D operation. Optical sensors are limited in their maximum detectable surface angle, usually defined by the numerical aperture of the chosen lens (Rahlves and Seewig, 2009). If the angle is exceeded no surface points (or erroneous surface points) are recorded. Also, widely used tactile profilometers are limited in their maximum angle. As the styli require a certain cone angle of 90 or $60^{\circ}$ and a tip radius of 2,5 or $10 \mu \mathrm{m}$ for stability and lateral stiffness, high surface angles lead to a wandering of the assumed contact point with resulting measurement deviation until eventually an unintentional flank collision occurs (Lonardo et al., 1996, ISO 3274:1996). The only sensors fully capable of 3-D measurements are the class of micro-tactile sensors. As the applied probing elements have diameters of $15 \mu \mathrm{m}$ and higher, 


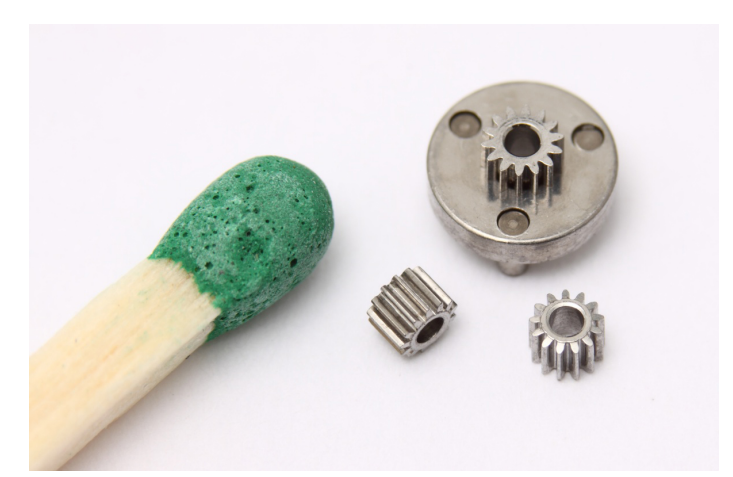

Figure 1. Gears of a micro-planetary drive. Diameter of the largest gear $4 \mathrm{~mm}$.

the accessibility of small structures and the lateral resolution is limited (UMAP Vision System, 2012; Weckenmann et al., 2004).

Apart from the sensor side progress in the field of coordinate measurement systems is also required. To realize the three-dimensional measurement of complex specimen in the nanometre order, the sensor systems (2-D or 3-D probe systems) should be additionally combined with appropriate mover systems (with possibly large movement range) for manipulating and measuring the relative position of the specimen to the probing tip. To fully utilize high accuracy sensors and to comply with Berndt's statements the positioning system must also have a resolution of up to sub-nanometre and an uncertainty of only a few nanometres. The classical coordinate measuring machines (CMMs) with moving probes, which commonly only fulfil the Abbe comparator principle in the $z$ axis, cannot meet this requirement (Weckenmann et al., 2004). For a three-dimensional measurement with the highest accuracy in the nanometre range, it is necessary to apply the Abbe comparator principle in all three coordinate axes. The current favourable way is to move the measurement object and to keep the probe systems in a fixed position, due to the fact that the workpiece carrier can be extended with a well-known and stable reference surface, e.g. a plane mirror, for a displacement measurement system. In this case, the probe system is used as a null indicator. To fulfil the Abbe principle in all axes the probing point lies in the intersection point of the three perpendicular measurement axes (Hausotte, 2011). Some of the mover systems like the positioning stage of the nanomeasuring machine SIOS NMM-1 already fulfil these requirements (Hausotte, 2011; Hausotte et al., 2009; Schott et al., 2013).

As sidewalls of workpieces or other 3-D structures are at present only measureable with tactile sensors, suitable measurement machines to operate them are required. In particular, a control system with various scan functions is necessary. A measurement task using a 2-D or 3-D sensitive probing system requires a predefined probing direction. Alternatively, the direction should be calculated in real time during the scan process for free-form scans and requires corresponding scan routines (Hausotte et al., 2009; Schott et al., 2013).

\section{Development of a 3-D nanometre measurement system}

To address the needs of modern measurement tasks, research has been conducted at the Friedrich-Alexander-Universität Erlangen-Nürnberg (FAU). Derived from the electrical probing interaction of scanning tunnelling microscopes (STM), a sub-nanometre resolving 3-D sensor was developed and combined with the nanopositioning and nanomeasuring machine NMM-1 to perform measurements on electrically conductive workpieces.

Scanning tunnelling microscopes were initially demonstrated by Binnig et al. (1982) and are based on the quantum tunnelling effect. By applying a voltage of a few millivolts between a conductive workpiece and a conductive sharp needle, a current signal of a few nanoamperes can be detected if the gap between both conductive partners is in the nanometre range. Due to the quantum tunnelling effect, electrons can pass the isolation barrier between both electrodes and contribute to an exponentially increasing current with a decreasing distance between the electrodes. The scanning tunnelling microscope easily allows for sub-nanometre resolution in the working direction and also in the lateral direction if the tip is sharp enough. The microscope, as in all scanning probe microscopes, is limited to flat surfaces due to the shape of the tip and the restricted working range of the positioning piezos.

At FAU the working principle was extended to three dimensions by using, instead of a needle-like tip, a metallic sphere as a sensor electrode allowing a probing on any part of the sphere (Weckenmann and Hoffmann, 2007; Hoffmann and Schuler, 2011). Apart from the gained 3-D capability, the application of probing spheres increases the area of electrical interaction between probe and workpiece, especially when sphere radii in the range from micro-metres to half a millimetre are applied. This way with the downturn of lowered lateral resolution, the vertical working range in surface direction increases up to $200 \mathrm{~nm}$ depending on the alloy of the sphere and workpiece as well as their surface roughness. The sensor characteristics and further aspects of the near-field interaction and involved effects were investigated in the works of Hoffmann (2009). The benefits are a force-free probing interaction without the danger of workpiece damage and an isotropic behaviour in all directions. Figure 2 shows the basic application and the resulting exponential characteristic curve if the sphere approaches the surface. Figure 3 displays the schematic of the surrounding set-up and the information flow. A precision voltage source is connected to the sensor electrode enabling the sensor operation. The distance-related current flow over the air gap of some nanometres is measured with a femtoampere amplifier with variable amplification and converted into a voltage signal. Usual amplification factors 

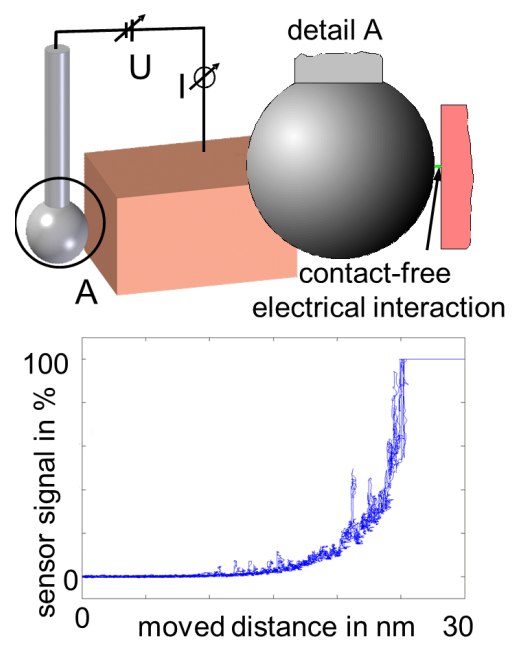

Figure 2. General set-up and characteristic of the electrical 3-D sensor.

range from 0.1 to $10 \mathrm{VnA}^{-1}$. As the amplifier's output voltage is limited to $\pm 10 \mathrm{~V}$, the amplification factor also influences the measurement range and is to be chosen in accordance with the desired set point. The characteristic in Fig. 2 displays this signal clipping at $100 \%$ sensor signal equivalent to $10 \mathrm{~V}$. The amplified signal is afterwards conditioned to match the input specifications of the connected positioning system, described in the following paragraph.

To make full use of the sensor's capabilities it was integrated in a nanopositioning and nanomeasuring system NMM-1 (Hausotte, 2011; Hausotte et al., 2012; Schott et al., 2013). The NMM-1 features a positioning and measuring volume of $25 \mathrm{~mm} \times 25 \mathrm{~mm} \times 5 \mathrm{~mm}$ and increases the sensor's measurement range equivalently. A corner mirror which is positioned by a three-axis drive system, is used for carrying the object to be measured (see Fig. 4). The accurate reference coordinate system is defined by the corner mirror and the position of the corner mirror is measured with three homodyne interferometers supplied by frequency stabilized lasers (Hausotte et al., 2011). The measuring axes intersect at the point where the sensor tip is situated. This set-up fulfils the Abbe comparator principle in three axes and the probe system acts in this case mostly only as a null indicator. The interferometer measurements are used for a closed-loop position control of the corner mirror. This allows for the compensation of the linear stage's guide path errors. By using two optical autocollimators in $x$ and $y$ axes, angular deviations of the guide system are also measured on the corner mirror. An additional angular control system compensates the angular errors using the four $z$ axis drives. The system provides a reliable measurement resolution of $0.1 \mathrm{~nm}$ with the smallest quantisation steps of $0.02 \mathrm{~nm}$. The positioning uncertainty is less than $10 \mathrm{~nm}$ for the entire measurement range.

The NMM-1 offers a simple integration of different sensor systems with its set of analogue inputs. The basic integration procedure is as follows: the voltage outputs of a sensor are recorded during surface approach and third-order polynomials are fitted into the data. The polynomials are applied in the machine's position control circuit to keep the sensor in its chosen working point of the calculated probing interaction signal (e.g. magnitude of probing force vector) (Hausotte, 2011). During a scanning operation with probing in vertical direction, the workpiece is moved (e.g. laterally) and surface height changes result in a change of the sensor signals. The position control circuit continually adjusts the workpiece carrier's height position to keep the configured set point. With the initial control design of the NMM-1 a sensor (1-D) was only used for the height control which limited the application to surfaces with a low curvature. With the recent developments of the NMM-1, a probing and position control via the sensor signal can be performed in all three spatial directions. Resulting possible scan routines are described in Sect. 5. Basically, the position control circuit regulates the distance sensor workpiece in the direction of the probing vector. The vector is either fixed like in the case of an optical point sensor which only operates in beam direction or the vector results from 3-D probing systems like, e.g. a micro-tactile system that outputs its deflection in three spatial directions.

\section{Application of the 3-D electrical probing system}

The electrical probing system has been used for 2-D as well as 3-D measurement tasks. As mentioned earlier the electrical probing system is capable of probing in any direction because of its spherical probe shape. In comparison to a microtactile probe, which outputs signals for the vector (magnitude and direction) of its deflection, the electrical sensor outputs only a magnitude signal independent of the geometric probing direction. As sophisticated scan commands (described in Sect. 5) require a 3-D probing force vector, mainly simple line scans and single point measurements are possible.

With the electrical sensor basically two different approaches are available. One method is to use a fixed probing vector like the height axis of the NMM-1. This method was used especially with the old control system of the NMM-1 and applied for measurements of flat surfaces and 3-D structures with limited curvature. Figure 5 shows a resulting application where the centre points of two metallic spheres on a ball bar are measured and their 3-D distance is measured.

The spheres' upper hemisphere can be measured nearly to their equators, but with increasing difference between probing vector and surface vector the stability of the position control system drops as the sensor characteristic changes. Furthermore, with the probing vector differing from the surface vector the form of the probing element has to be compensated as the actual probing point wanders from its assumed position. 


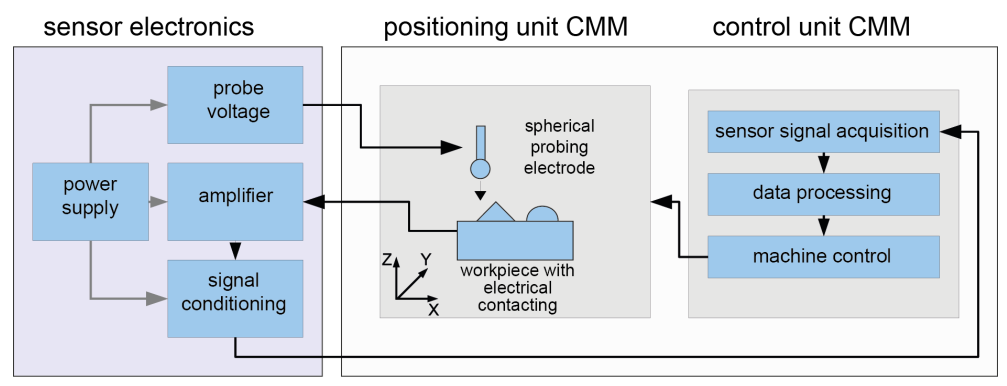

Figure 3. Information flow in the measurement set-up.

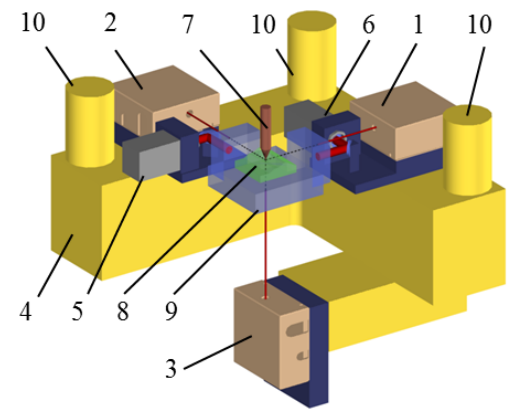

Figure 4. Principle design of the nanomeasuring machine: (1) $x$ interferometer, (2) $y$ interferometer, (3) $z$ interferometer, (4) metrology frame made of Zerodur, (5) roll and yaw angular sensor, (6) pitch and yaw angular sensor, (7) surface-sensing probe, (8) sample, (9) mirror corner, and (10) fixing points for probe system.

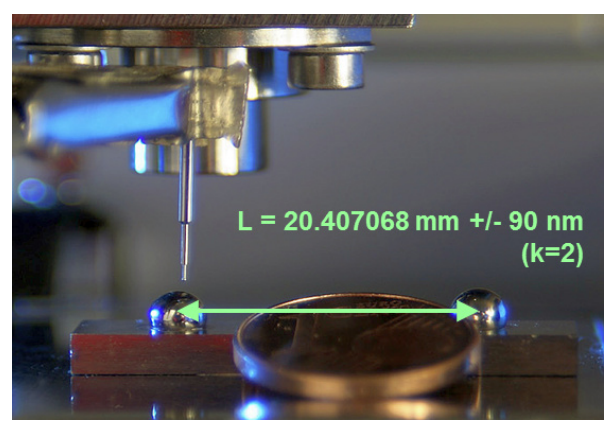

Figure 5. Distance measurement on a ball bar.

Another exemplary 3-D measurement task is displayed with Fig. 6 where the structures on a cutting insert are inspected.

With the newer control system a programmable probing vector is also possible. The probing vector is precalculated to match the local surface angle either derived from a first measurement pass or from a computer aided design (CAD) model. A standard measurement task which was performed with the electrical sensor would be the thickness calibration of a gauge block where the probing is performed in a lateral direction. Another example would be the point-wise mea-

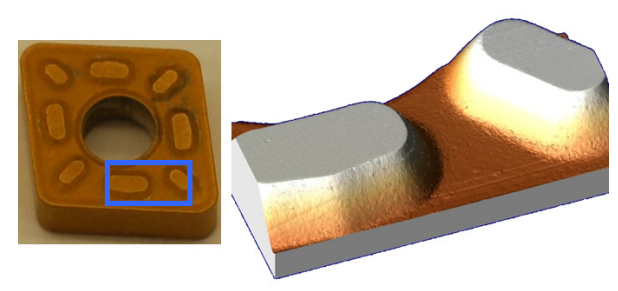

Figure 6. Surface measurement on a cutting insert, area $6 \mathrm{~mm} \times 3 \mathrm{~mm}$.

surement on a sphere where, with the use of a stylus tree (Hoffmann and Schuler, 2011), the full surface, also below the equator, can be measured.

The disadvantage of this 3-D probing system is, similar to micro-tactile systems, the limited lateral resolution on the surface. Only with a very small sphere or very sharp tip can high lateral resolutions or high structure resolutions be achieved, but this again leads to possible shaft collisions and limited 3-D capability.

\section{Extension of measurable surface angles}

As mentioned in the introduction most sensors show a limited measurable surface angle or at least high measurement deviation for measurements with high inclinations to the normal vector of the surface. Therefore, a method to achieve 3-D capability with 1-D probes was developed at the FAU which can be applied to many sensors. The aim was to increase the effectively measurable surface angle and to reduce measurement deviation by keeping a sensor in its optimal working angle during the measurement. This sensor tilting principle was planned and simulated in theory and the principle was afterwards transferred into a hardware prototype where the suggested principle and its effectiveness were demonstrated (Weckenmann et al., 2012; Schuler, 2013; Schuler et al., 2014). The simulation calculated the occurring measurement deviation of a tactile profilometer on an arbitrary surface and the effect of the tilting was investigated. A main task was the development of the control theory to calculate the sensor's optimal tilt angle at a certain surface position during a surface measurement. Different strategies to calculate 

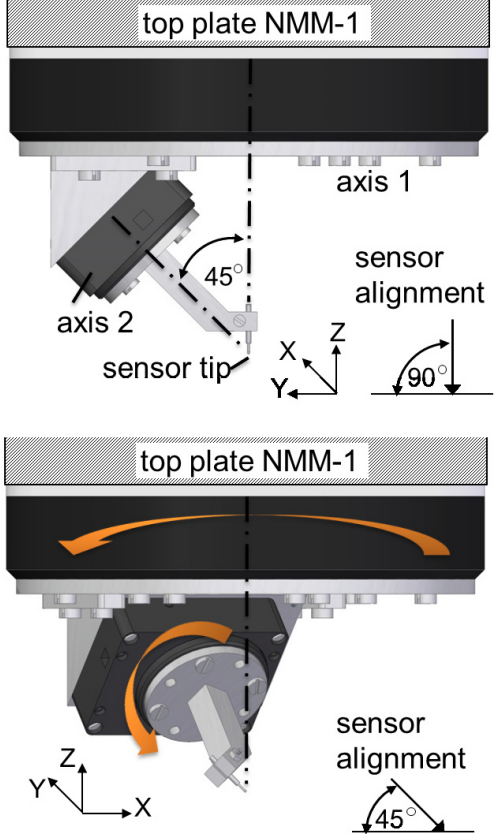

Figure 7. Schematic of the rotary kinematic chain, operated at $90^{\circ}$ and $45^{\circ}$ sensor alignment.

the sequence of tilt angles were investigated. With knowledge based strategies using, e.g. a CAD model, the ideal angles can be calculated beforehand; therefore, with analysing strategies a first scan pass with fixed sensor alignment delivers the calculation basis for a second pass, and with predicting strategies the surface angle is extrapolated during the measurement from already acquired surface points. A series of test cases were simulated and generally a sensor tilt of already $10^{\circ}$ could significantly lower the measurement deviation caused by operating the sensor out of its optimal working angle. The simulation results also created the input data for the practical realisation. Different kinematic chains were calculated and compared with the aim that for an effective operation, the probing point of the sensor should not deviate more than $\pm 500 \mathrm{~nm}$ after tilting. In result a kinematic system was developed to rotate a 1-D sensor around its probing point in space with a maximum rotation angle of $\pm 90^{\circ}$ in 2 degrees of rotation. During a profile measurement this allows for the continuous angular realignment to keep a sensor perpendicular to the workpiece surface (sensor axis parallel to the normal vector of the surface).

As a testing platform for the prototype the NMM-1 was used mounting the rotary kinematic chain above the workpiece carrier. Figure 7 shows a drawing of the developed rotation system and its basic operation principle.

The set-up consists of two stacked rotary tables. The first one with a diameter of $100 \mathrm{~mm}$ is a direct-driven stage and the second one with $30 \mathrm{~mm}$ diameter uses a self-locking piezo drive. The second axis is mounted below the first axis under an angle of $45^{\circ}$ and carries a sensor tip in a sensor

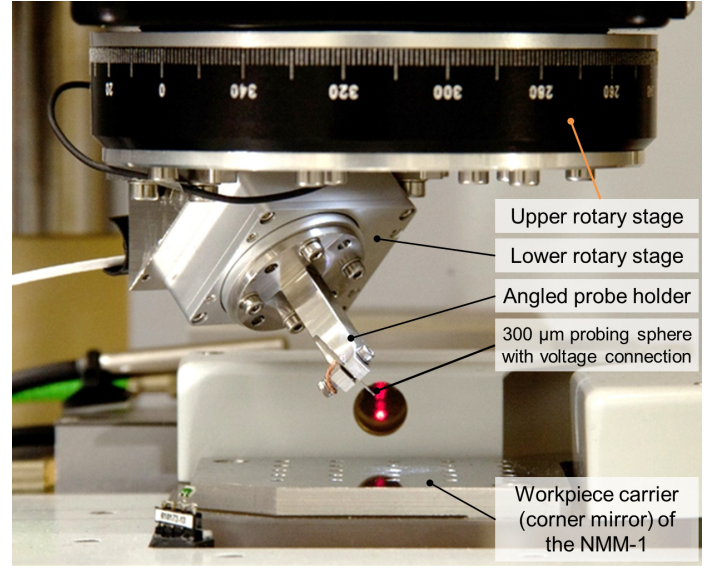

Figure 8. Kinematic chain installed into the NMM-1 with the electrical near-field sensor.

holder again under a $45^{\circ}$ angle. In a default orientation the sensor is held perpendicular to the $X-Y$ layer of the NMM1 's workpiece carrier. Both rotation axes are aligned in a way that their centre axes and the sensor probing point virtually intersect, apart from alignment deviations. By turning the lower axis, the sensor revolves around its working point and turns into the $X-Y$ plane of the NMM-1, resulting in rotation components around $X$ and $Y$. With the additional movement of the upper rotation stage, the $X$ and $Y$ components are translated into each other and a desired combination can be achieved, e.g. a $45^{\circ}$ rotation around the $y$ axis in Fig. 7 (lower panel). The controllers of both axes are connected to a host computer and are integrated into the sequence control based on MATLAB which controls the NMM-1 and also realizes the tilting strategy and performs coordinate transformations. To allow for a fast translation of a required Cartesian sensor tilt angle into the corresponding angular positions of the rotary stages, a look up table is also included. Figure 8 displays the finished set-up.

As mentioned before to ensure a feasible operation of this kinematic for micro- and nanometrology, the residual deviation of the sensor's working point after rotation has to be below $\pm 500 \mathrm{~nm}$. As the applied rotation axes can show systematic position errors in the micro-metre range and possible alignment errors also have to be respected, an in situ calibration procedure was created. It relies on the positioning ability of the NMM-1 and uses for best effectiveness the already described electrical 3-D sensor.

The calibration procedure implements the so-called empirical qualification from ISO 10360-5:2010 for articulating probing systems (ISO 10360-5:2010). On the workpiece carrier a calibration sphere is mounted and its centre serves as a reference point being measured under different sensor angles. With the nanometre resolving electrical sensor and its ability to work with spherical probes, the full upper hemisphere of the calibration sphere can be measured, but the 


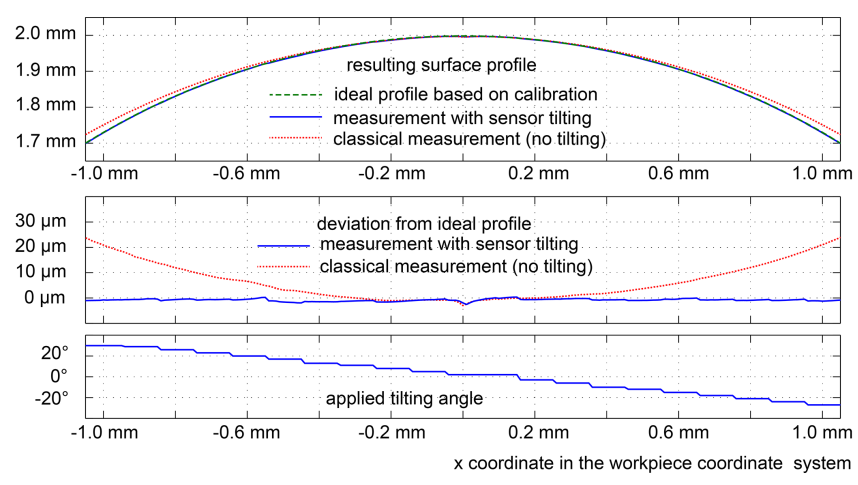

Figure 9. Measurement results with and without sensor tilting on a $4 \mathrm{~mm}$ sphere.

procedure can also be used with other sensors, e.g. a tactile profilometer or optical point probe. The resulting centre points allow the calculation of the sensor's probing point in relation to the reference sphere. The movement vectors of the sensor caused by rotation are stored in a compensation field and are compensated by the NMM-1's positioning abilities. With this approach compensating detectable systematic error components the remaining residual position error of the kinematic chain was identified. The range of the sensor position over the calibrated angular range, defined by the nonsystematic error components and the non-compensated systematic components, was below $140 \mathrm{~nm}$ in the worst Cartesian direction. This parameter is also referred to as $P_{\mathrm{LTE}}$ in ISO 10360-5:2010. Compared to the initial requirement of the error range being smaller than $1 \mu \mathrm{m}( \pm 500 \mathrm{~nm})$, the realized kinematic chain successfully enables the investigation of the sensor tilting principle in practice.

An exemplary measurement is shown in Fig. 9 comparing measurements on a $4 \mathrm{~mm}$ sphere, all conducted with the electrical sensor and a probing tip of $150 \mu \mathrm{m}$ curvature radius. The dotted red line in upper part of Fig. 9 shows the results of a regular measurement with the electrical sensor and a vertical sensor alignment. Here the electrical sensor is operated like a profilometer meaning a fixed probing vector and a fixed contact point on the bottom of the tip is assumed. The surface profile is superimposed with the tip shape leading with increasing surface angles to increasing measurement deviation (see middle part of Fig. 9). This convolution can be compensated to a certain degree, especially well on spheres, but the deconvolution is limited on more complex surfaces (Keller, 1991). In comparison, an optical sensor would only be able to record the surface to a certain degree and would get erroneous or no data if the angular limit is exceeded.

With the shown sensor tilting in contrast, the sensor can be kept in its optimal angle, the contact point between tip and surface does not wander and the deviation is avoided. In the middle of the recorded profile in Fig. 9 both curves coincide as the regular measurement operates in its optimal working angle. With increasing distance from the sphere's pole the deviation increases and the benefit of the tilting principle is visible. Basically with the current kinematic chain, the effectively measurable surface angle of an installed sensor is increased to more than $\pm 90^{\circ}$.

The electrical near-field 3-D sensor was used for the calibration procedure and to demonstrate the difference between a fixed and a tilted operation. With its 3-D capabilities and the NMM-1's 3-D control unit, a tilting would naturally not be required as the spherical probe can interact in any direction if the probing vectors are chosen appropriately as described in Sect. 3. With a tiltable 1-D probe and very sharp tip a high lateral resolutions or high structure resolutions can be achieved. Current research covers therefore the transfer of this sensor tilting principle to other sensors like optical 1-D sensors.

\section{3-D measurement routines of nanocoordinate measurement machines}

Apart from developments on the sensor side progress on the side of nanocoordinate measurement machines can also be reported. The current firmware and 3-D position control unit of the NMM-1 provides a complete 3-D control system based on the I++ DME (Inspection plus plus for Dimensional Measurement Equipment) specification for implementing point and scan measurement instructions (Hausotte, 2011; Hausotte et al., 2009; Schott et al., 2013).

Basically the sensors can be operated in an open-loop or a closed-loop mode. Simple open-loop scan commands use only the machine position as the input and the probe is moved along a predefined trajectory across the workpiece to record a profile. If a threshold is specified in this situation where the sensor would exceed its working range, the scan trajectory can be modified during the scan by moving away or towards from the surface. The scanning can also be conducted in a continuous closed-loop operation by maintaining a constant deflection or probing force at all times during the scanning, e.g. along a line, around a circular path or as a helix. Furthermore with a so-called "dodge scan" an open- and a closed-loop scan can be combined. An automatic switching between both the methods is thus feasible in order to improve the measurement speed and accuracy. If the sensor exceeds its working range the position control is switched to closedloop and evades the obstacle, afterwards it switches back to the open-loop operation. With a two- or three-dimensional sensitive probing system, a measurement operation with the free-form scan is possible. In this case, the probing direction does not have to be predefined. It only depends on the form of the sample surface and is calculated from the deflection vector of the probe.

With different newly implemented scan techniques and various instructions, especially the three-dimensional measurements of micro-structures can be flexibly operated. They are intended to be used with 3-D sensors with a strong focus 


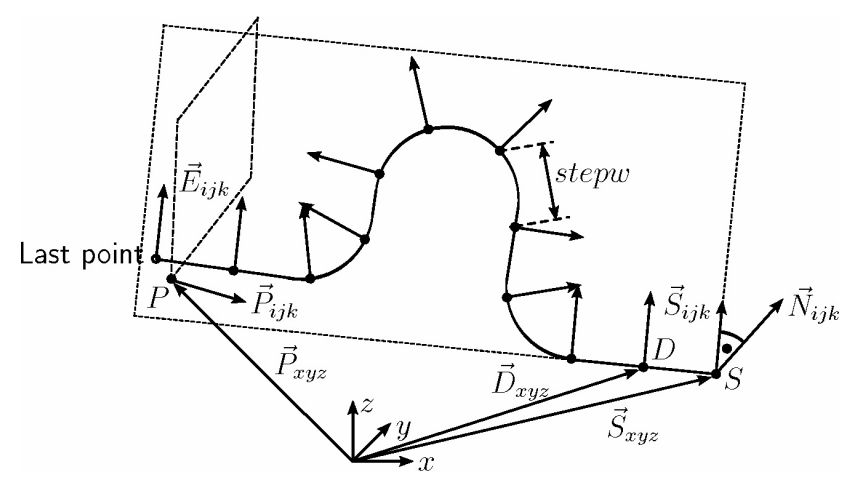

Figure 10. Principle and parameters of free-form scan function "ScanInPlaneEndIsPlane".
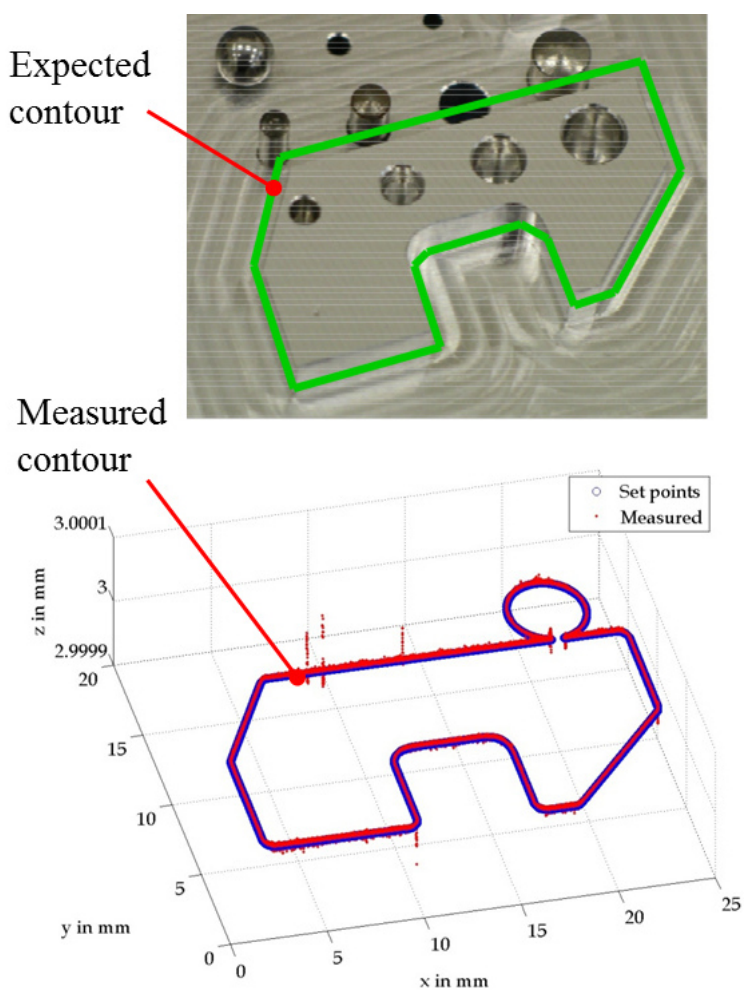

Figure 11. Free-form scan on a sample structure.

on 3-D sensitive tactile micro-probes featuring small probing spheres of down to $15 \mu \mathrm{m}$ diameter. Such micro-probes are commonly designed to detect the probing force as well as to determine the probing direction (Balzer et al., 2011). This type of probe is advantageous for a free-form scan of workpieces with high aspect ratios. In this case the probing vector between probing tip and surface is directly measured and an optimal probing direction can be easily calculated from the detected force vector in real time. It only depends on the form of the sample surface and does not have to be predefined.

As an example for the routines developed for 3-D probing systems, the free-form scan instruction "ScanInPlaneEndIs-

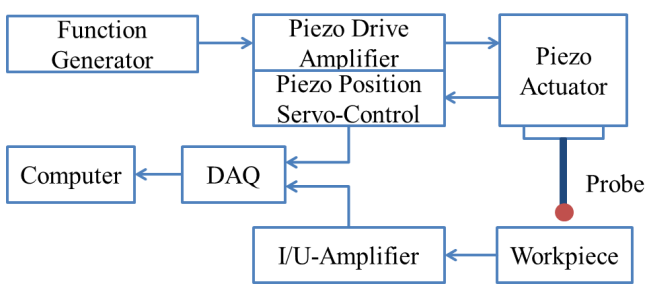

Figure 12. Block diagram indicating key components of the measurement system.

Plane" is shown in Fig. 10. At first a surface approach is executed and the start point $S_{x y z}$ on the surface is probed. The controller attempts to follow the contour in a plane defined by $N_{i j k}$ with given scan speed, and samples the data points with a constant point distance (stepw). The scan direction is calculated from the probing force vector, measured by the tactile probe and its magnitude, and it is calculated with the plane definition. The probing force magnitude is controlled while the deflections and force magnitude are monitored. The scan terminates when the last measured point exceeds the defined end plane.

Figure 11 illustrates a free-form scan of a sample structure using "ScanInPlaneEndIsPlane". A conventional tactile probe with a diameter of $1 \mathrm{~mm}$ was used to demonstrate the function. The gap between the biggest bolt and the contour was too slim for the probe sphere to run through. Thus, the controller has chosen another path, around the bolt, to continue the scanning. This prevents expensive probe damage especially on micro-parts where the operator can no longer track the probe system only with his eyes. At each point of the contour the normal vector of the contact surface must be calculated by the NMM- 1 controller in order to determine, whether the scan direction should be maintained or with a small change, or even turn sharp to totally another direction.

To benefit from those flexible instructions for free-form scans, it is planned to extend the electrical near-field sensor with capabilities to directly detect its contact direction within an ongoing research project. When the magnitude and the direction of the contact vector are known, the elaborate measurement commands for free-form surfaces can be used.

\section{3-D vector extension of the electrical probing system}

To address this extension of the electrical probing system's abilities, a superimposed mechanical oscillation is investigated. By using a metallic sphere as a sensor electrode a probing on any part of the sphere is possible and the sensor is hence sensitive in all directions in the measuring space. But differing from the force signal of a tactile probe, the electrical signal is only a scalar, and the direction information has to be gathered otherwise. If a mechanical circular movement is applied the probing direction can be derived from the 


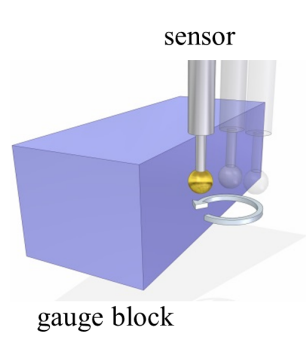

(a)

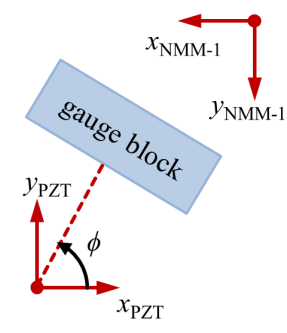

(b)
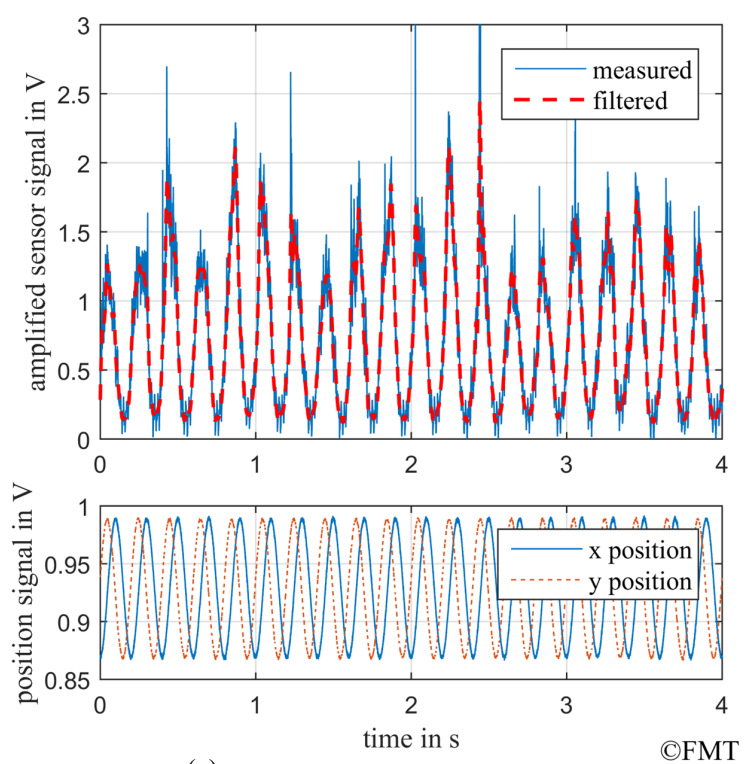

(c)

Figure 13. (a) Arrangement of the measurement system; (b) top view with the coordinate system of the piezo actuator, $\phi$ : to be determined angle; (c) measurement results.

relation between sensor signal and circular sensor position (Ogura and Okazaki, 2002). The frequency of the circular movement and the speed of the vector calculation should be high enough to deliver the NMM-1 a 3-D probing vector during the scanning motion.

Figure 12 shows the configuration of a first measurement set-up. The probe is mounted on a 3-D movable, precise piezo scanner consisting of a function generator, a piezodrive amplifier, a piezo-position-servo control, and a piezo actuator. As sine and cosine wave signals generated from a function generator are synchronously applied to the actuators, the probe is moving on a circular path of some nanometres. When the path is within the working range of the electrical sensor, a periodic tunnelling current signal will be measured. A data acquisition box (DAQ) can be used to register the data of the actuator position and the amplified current signals synchronously. The NMM-1 is used for a positioning of the workpiece in to the working range of the probe.

As an example a side wall scan of a gauge block with a spherical probe is shown in Fig. 13a. The probe moved circularly in the $X-Y$ plane around the origin of the piezo actuator coordinate system. Depending on the accuracy of the mechanical adjustment/integration of the sensor system, the coordinate system of the actuator is parallel to that of the nanomeasuring machine. The results of the preliminary setup still indicate drift and vibration effects and the mechanical set-up is therefore being revised. With the planned connection to the NMM-1's position control, the results can be further improved. Nevertheless, with the help of low-pass filtering and Fourier analysis the characteristics of the signals and the resulting probing vector can be determined. The dif- ference between the phase shifts of the sensor signal and the $x$ position signal is consistent with the angle $\phi$, as specified in Fig. 13b. In this condition the probing vector $(\cos \phi, \sin \phi)$ is consequently identified in the piezo actuator coordinate system.

The further steps involve the set-up of a separate control unit based on a digital signal processor for real-time calculations. This unit will substitute the function generator and the data acquisition. It will be able to create the circular movement path on all three axes and will forward the calculated proving vector to the NMM-1. With the connection to the NMM-1 the position control based on the sensor signal will be realized and the investigations can continue.

\section{Conclusions}

Driven by modern measurement tasks requiring nanometre resolving sensors with 3-D capability and appropriate nanopositioning systems with suitable scan routines, developments to address these problems have been demonstrated.

To meet these requirements a working principle known from scanning probe microscopes was adapted realising subnanometre sensor resolution with a contact free 3-D probing capability. In combination with a nanopositioning system type NMM-1, an operation, such as a 1-D sensor or a 3-D sensor, is possible allowing even the probing of sidewalls and undercuts. Current developments focus on directly detecting the contact vector between sensor and workpiece so more elaborate scan routines can be applied. For this aim research is being conducted to superimpose a circular movement to a 
3-D capable probing element and to derive the contact vector from the relation between the circular position and signal.

Apart from the electrical sensor another approach to gain 3-D ability was demonstrated based on the tracking of a 1-D sensor's operation angle during a measurement. A rotary kinematic chain turns a sensor around its working point and keeps its orientation perpendicular to the surface. This effectively reduces measurement deviation on sloped surfaces and extends the accessible surface angle to $\pm 90^{\circ}$ in 2 degrees of freedom. Paired with an online compensation of systematic position errors the principle was successfully brought to application.

On the side of 3-D positioning systems progress also is reported with the new control system and scan routines of the nanopositioning and nanomeasurement system NMM-1. The routines allow for free-form scans on 3-D objects enabling the operator to make full use of 3-D sensors. Due to the decreased visibility of micro-parts for the operator and the always prevailing danger of damaging a micro-probe elaborate, scan routines are necessary.

Acknowledgements. The authors wish to thank all those colleagues at the FAU and the Ilmenau University of Technology who have contributed to these developments. The authors also thank the German Research Foundation (Deutsche Forschungsgemeinschaft, DFG). Section 4 is based on the research grant WE 918/32-1 conducted at the chair QFM (FAU) under the leadership of Albert Weckenmann. The current development of the electrical sensor is supported by research grant HA 5915/4-1.

Edited by: R. Schmitt

Reviewed by: two anonymous referees

\section{References}

Balzer, F. G., Hausotte, T., Dorozhovets, N., Manske, E. and Jäger, G.: Tactile 3-D microprobe system with exchangeable styli, Meas. Sci. Technol., 22, 094018, doi:10.1088/09570233/22/9/094018, 2011.

Berndt, G., Hultzsch, E., and Winhold, H.: Funktionstoleranz und Meßunsicherheit, Wissenschaftliche Zeitschrift der Technischen Universität Dresden, 17, 465-471, 1968.

Binnig, G., Rohrer, H., Gerber, C., and Weibel, E.: Surface Studies by Scanning Tunneling Microscopy, Phys. Rev. Lett., 49, 57-61, 1982.

Bissacco, G., Hansen, H. N., and Slunksy, J.: Modelling the cutting edge radius size effect for force prediction in micro milling, CIRP Ann.-Manuf. Techn., 57, 113-116, 2008.

Hansen, H. N., Carneiro, K., Haitjema, H., and De Chiffre, L.: Dimensional micro and nanometrology, CIRP Ann.-Manuf. Techn., $55,721-743,2006$.

Hausotte, T.: Nanopositionier- und Nanomessmaschinen - Geräte für hochpräzise makro- bis nanoskalige Oberflächen- und Koordinatenmessungen, Ilmenau, Techn. Univ. Habilitationsschrift. Pro Business, ISBN 978-3-86805-948-9, 2011.
Hausotte, T., Percle, B., Manske, E., Füßl, R., and Jäger, G.: Measuring value correction and uncertainty analysis for homodyne interferometers, Meas. Sci. Technol., 22, 094028, doi:10.1088/0957-0233/22/9/094028, 2011.

Hausotte, T., Balzer, F. G., Vorbringer-Dorozhovets, N., and Manske, E.: Surface and coordinate measurements with nanomeasuring machines, Int. J. Nanomanufacturing, 8, 467483, 2012.

Hausotte, T., Percle, B., and Jäger, G.: Advanced three-dimensional scan methods in the nanopositioning and nanomeasuring machine, Meas. Sci. Technol., 20, 084004, doi:10.1088/09570233/20/8/084004, 2009.

Hoffmann, J.: Elektrische Werkstückantastung für Nanometer aufgelöste Oberflächen- und Koordinatenmesstechnik, Berichte aus dem Lehrstuhl Qualitätsmanagement und Fertigungsmesstechnik, 2009/18, Universität Erlangen-Nürnberg, Dissertation, 2009.

Hoffmann, J. and Schuler, A.: Nanometer resolving coordinate metrology using electrical probing, Tech. Mess., 78, 142-149, 2011.

International Organization for Standardization: Geometrical Product Specifications (GPS) - Surface texture: Profile method Nominal characteristics of contact (stylus) instruments, ISO 3274:1996, 1996.

International Organization for Standardization: Geometrical product specifications (GPS) - acceptance and reverification tests for coordinate measuring machines (CMM): part 5. CMMs using single and multiple stylus contacting probing systems ISO 10360-5:2010, 2010.

Jiang, X. and Whitehouse, D. J.: Technological shifts in surface metrology, CIRP Ann.-Manuf. Techn., 61, 815-836, 2012.

Keller, D.: Reconstruction of STM and AFM images distorted by finite-sized tips, Surf. Sci., 253, 353-364, 1991.

Lonardo P. M., Trumpold, H., and De Chiffre, L.: Progress in 3-D Surface Microtopography Characterization, CIRP Ann.-Manuf. Techn., 45, 589-598, 1996.

Ogura, I. and Okazaki, Y.: Development of micro probe for microCMM, Proc. of ASPE 2002, 349-352, 2002.

Rahlves, M. and Seewig, J.: Optisches Messen Technischer Oberflächen - Messprinzipien und Begriffe, BeuthVerlag, Berlin, Germany, 2009.

Schott, W., Dontsov, D., Langlotz, E., and Manske, E.: Im Nanobereich - Multisensorsystem für Präzisionsmessungen und Kalibrierung, QZ Qualität und Zuverlässigkeit, 58, 42-43, 2013.

Schuler, A.: Erweiterung der Einsatzgrenzen von Sensoren für die Mikro- und Nanomesstechnik durch dynamische Sensornachführung unter Anwendung nanometeraufgelöster elektrischer Nahfeldwechselwirkung, Erlangen, Dissertation, Shaker Verlag, ISBN 978-3-8440-2299-5, 2013.

Schuler, A., Weckenmann, A., and Hausotte, T.: Setup and evaluation of a sensor tilting system for dimensional microand nanometrology, Meas. Sci. Technol., 25, 064010, doi:10.1088/0957-0233/25/6/064010, 2014

UMAP Vision System PR 1207(2), datasheet, Mitutoyo, Germany, 10 pp., 2012.

Weckenmann, A., Estler, T., Peggs, G., and McMurtry, D.: Probing systems in dimensional metrology, CIRP Ann.-Manuf. Techn., 53, 657-684, 2004. 
Weckenmann, A. and Hoffmann, J.: Long Range 3-D Scanning Tunnelling Microscopy, CIRP Ann.-Manuf. Techn., 56, 525528, 2007.

Weckenmann, A., Tan, Ö., and Hartmann, W.: Function Oriented Characterization for Surface Metrology, International Journal of Nanomanufacturing, 7, 517-527, 2011.
Weckenmann, A., Schuler, A., and Ngassam, R. J. B.: Enhanced measurement of steep surfaces by slope-adapted sensor tilting, Meas. Sci. Technol., 23, 074007, doi:10.1088/09570233/23/7/074007, 2012. 